\title{
Dual chiral density waves in nuclear matter
}

\author{
Hiroaki Abuki ${ }^{1, *}$, Yusuke Takeda ${ }^{2, * *}$, and Masayasu Harada ${ }^{2, * * *}$ \\ ${ }^{1}$ Department of Education, Aichi University of Education, 1 Hirosawa, Kariya 448-8542, Japan \\ ${ }^{2}$ Department of Physics, Nagoya University, Nagoya, 464-8602, Japan
}

\begin{abstract}
We study inhomogeneous chiral phases in nuclear matter using a hadronic model with the parity doublet structure. With an extended ansatz for the dual chiral density wave off the chiral limit, we numerically determine the phase structure. A new type of dual chiral density wave where the condensate has nonvanishing space average is confirmed and it comes to occupy a wide range of low density region as the chiral invariant mass parameter is lowered.
\end{abstract}

\section{Introduction}

The chiral symmetry breaking serves as a key ingredient to understand the phase structure of QCD, since it is responsible for the mass generation of hadrons as well as the mass splittings of chiral partners. The chiral symmetry is expected to restore at sufficiently high baryon density. An interesting possibility opens up when one allows the chiral condensate to vary in space; Nakano and Tatsumi demonstrated that the symmetry restoration may take place via several steps [1]; going up in density from the vacuum, the system first goes into an intriguing state named as dual chiral density wave (DCDW), that is, a particular type of inhomogeneous chiral phases, making a spiral in the $\left(\sigma_{0}, \pi_{0}\right)$ chiral plane along $z$ direction.

There are number of approaches to chiral inhomogeneous phases [2]. These include; the mean-field approximation [3-6], the Ginzburg-Landau expansion [7-10], a self-consistent mean-field approach [11]. These are based on quark-based models such as an NJL-type model. One of the advantages is that these models are capable of realizing the QCD vacuum properties as well as the color-flavor locked phase of quark matter which is known to be the densest phase of QCD. But there is also a disadvantage, that is, the lack of the ability to reproduce properties of nuclear matter at saturation point, namely, the QCD phase next to the vacuum phase being followed right after the liquid-gas phase transition.

We here report on our recent work on this topic [12] where we adopted a hadronic model with parity doublet picture (mirror assignment) [13, 14], with vector mesons included in a manner guided by the hidden local symmetry $[15,16]$. We first extend the ansatz for the DCDW phase so as to take into account the effect of the explicit symmetry breaking. The extended ansatz smoothly interpolates between the DCDW phase and a nearly symmetryrestored phase. With this setup, we construct the effective potential by diagonalizing the Bogoliubov-de Gennes (BdG) Hamiltonian for nucleons, and determine phases via numerically minimizing the potential. Our main finding is the emergence of another type of DCDW phase which occupies the lower density region according to the value of chiral invariant mass.

\footnotetext{
*e-mail: abuki@auecc.aichi-edu.ac.jp

**e-mail: yusuketakeda4370@gmail.com

***e-mail: harada.masayasu@ nagoya-u.jp
} 


\section{Model}

In our analysis, we introduce $N^{*}(1535)$ as the chiral partner to the ordinary nucleon based on the parity doublet structure. Along the line described in [17], we construct a relativistic mean field model to describe nuclear matter, which includes the scalar $(\sigma)$, pseudoscalar $(\pi)$ mesons, and also the vector $(\omega)$ meson within the unitary gauge of the hidden local symmetry.

The pure mesonic part for the Lagrangian is then given by

$$
\begin{aligned}
\mathcal{L}_{\text {mes. }}= & \frac{m_{\omega}^{2}}{2} \omega_{\mu} \omega^{\mu}-\frac{1}{4} \omega_{\mu \nu} \omega^{\mu v}+\frac{1}{2} \partial_{\mu} \sigma \partial^{\mu} \sigma+\frac{1}{2} \partial_{\mu} \pi \partial^{\mu} \pi \\
& +\frac{\bar{\mu}^{2}}{2}\left(\sigma^{2}+\pi^{2}\right)-\frac{\lambda_{4}}{4}\left(\sigma^{2}+\pi^{2}\right)^{2}+\frac{\lambda_{6}}{6}\left(\sigma^{2}+\pi^{2}\right)^{3}-f_{\pi} m_{\pi}^{2} \sigma,
\end{aligned}
$$

where $\bar{\mu}^{2}, \lambda_{4}$ and $\lambda_{6}$ are model parameters. $\omega_{\mu}$ and $\omega_{\mu \nu}=\partial_{\mu} \omega_{v}-\partial_{v} \omega_{\mu}$ are the field and field strength for $\omega$ meson, which are singlet under the chiral transformation. The mass and the decay constant of the pion, as well as the mass of omega meson, are to be set to physical values $m_{\pi}=139 \mathrm{MeV}, f_{\pi}=92.2 \mathrm{MeV}$, and $m_{\omega}=783 \mathrm{MeV}$, respectively.

In the present model based on the parity doublet structure, the transformation properties of the positive and negative parity nucleon fields are given by

$$
\begin{aligned}
& \psi_{1 r} \rightarrow g_{R} \psi_{1 r}, \quad \psi_{2 r} \rightarrow g_{L} \psi_{2 r}, \\
& \psi_{1 l} \rightarrow g_{L} \psi_{1 l}, \quad \psi_{2 l} \rightarrow g_{R} \psi_{2 l},
\end{aligned}
$$

where $g_{R}\left(g_{L}\right)$ is an element of $\mathrm{SU}(2)_{R}\left(\mathrm{SU}(2)_{L}\right)$ chiral symmetry group, and $\psi_{1 r}$ and $\psi_{2 r}\left(\psi_{1 l}\right.$ and $\psi_{2 l}$ ) are the right-handed (left-handed) fields. Based on the transformation properties, the Lagrangian relevant for nucleons is expressed as [17]

$$
\begin{aligned}
\mathcal{L}_{\mathrm{N}-\mathrm{mes}}= & \bar{\psi}_{1}\left(i \not \supset+\mu_{B}-g_{\omega} \phi\right) \psi_{1}+\bar{\psi}_{2}\left(i \not \partial+\mu_{B}-g_{\omega} \phi\right) \psi_{2}-m_{0}\left(\bar{\psi}_{2} \gamma_{5} \psi_{1}-\bar{\psi}_{1} \gamma_{5} \psi_{2}\right) \\
& -g_{1} \bar{\psi}_{1}\left(\sigma+i \gamma_{5} \pi \cdot \tau\right) \psi_{1}-g_{2} \bar{\psi}_{2}\left(\sigma-i \gamma_{5} \pi \cdot \tau\right) \psi_{2}
\end{aligned}
$$

where the baryon chemical potential is included via $\mu_{B}=\mu_{B} \gamma_{0} . \tau=\left(\tau^{1}, \tau^{2}, \tau^{3}\right)$ is a set of Pauli matrices, $g_{1}, g_{2}$ and $g_{\omega}$ are the coupling constants. $m_{0}$ is the chiral invariant mass parameter which survives as a nonvanishing nucleon mass even when the chiral symmetry is restored.

In the present analysis, we adopt the following extended DCDW ansatz, $\sigma=\delta \sigma+$ $\sigma_{0} \cos (2 f z), \pi_{3}=\sigma_{0} \sin (2 f z)$, that reads in the complex representation:

$$
\langle\sigma+i \pi \cdot \tau\rangle=\delta \sigma+\sigma_{0} e^{2 i f z \tau^{3}} \equiv M(z)
$$

where $\delta \sigma, \sigma_{0}$ and $f$ are variational parameters with dimension one. Space independent part $\delta \sigma$ accommodates the possibility that the space average of DCDW condensate would get nonvanishing shift into $\sigma$-direction due to the explicit chiral symmetry breaking. In the meanfield approximation, the nucleon contribution to the effective potential can be written as

$$
\Omega_{N}=\frac{i}{V_{4}} \operatorname{Tr} \log \left(i \partial_{0}-\left(\mathcal{H}(z)-\mu_{B}^{*}\right)\right),
$$

where $V_{4}$ is the space-time volume and $\mathcal{H}(z)$ is the single particle Bogoliubov-de Gennes (BdG) Hamiltonian defined in the space of fermion bispinor $\psi=\left(\psi_{1}, \psi_{2}\right)$ as

$$
\mathcal{H}=\left(\begin{array}{cc}
i \gamma^{0} \gamma \cdot \nabla+g_{1} \gamma^{0}\left(M(z)^{\dagger} P_{L}+M(z) P_{R}\right) & m_{0} \gamma^{0}\left(P_{R}-P_{L}\right) \\
m_{0} \gamma^{0}\left(P_{L}-P_{R}\right) & i \gamma^{0} \gamma \cdot \nabla+g_{2} \gamma^{0}\left(M(z) P_{L}+M(z)^{\dagger} P_{R}\right)
\end{array}\right),
$$


where $P_{R(L)}=\frac{1 \pm \gamma_{5}}{2}$ is the chirality projector. This is nothing but the Dirac Hamiltonian in the presence of a periodic potential field $M(z)(=M(z+\lambda)$ with a wavelength $\lambda=\pi / f)$. Then the functional trace in Eq. (5) can be evaluated by finding eigenvalues of the operator $\mathcal{H}(z)$ [18]. The eigenvalue has a discrete label as well as continuous three-momentum $\mathbf{p}$ in addition to internal quantum numbers; This is because of the Bloch theorem which states that the eigenfunctions in the presence of a periodic potential are the plane waves distorted by periodic functions. To be specific, we decompose the bispinor as

$$
\psi(\mathbf{x})=\sum_{\ell=-\infty}^{\infty} \sum_{\mathbf{p}_{\perp}} \sum_{\left|p_{z}\right| \leq f} e^{i \mathbf{p} \cdot \mathbf{x}+i \boldsymbol{K}_{\ell} \cdot \mathbf{x}} \psi_{\mathbf{p}, \ell},
$$

where $\boldsymbol{K}_{\ell}=(0,0,2 f \ell)$ is the reciprocal lattice vector. Moving on to the quasimomentum base $\left\{\psi_{\mathbf{p}, \ell}\right\}$, the BdG Hamiltonian becomes block-diagonalized, $H_{\ell \ell^{\prime}}(\mathbf{p})$. Since the isospin remains a good quantum number, we can simply double the proton contribution in the full effective potential. Then omitting the antiprotons which would not contribute at zero temperature, and diagonalizing $H_{\ell \ell^{\prime}}(\mathbf{p})$ results in an infinite tower of eigenvalues at each $\mathbf{p}$, which repeatedly appears for every Brillouin Zone (BZ), $\mathbf{p} \rightarrow \mathbf{p}+\boldsymbol{K}_{\ell}(\ell=\cdots,-1,0,1, \cdots)$ :

$$
\sum_{\ell^{\prime}} H_{\ell \ell^{\prime}}(\mathbf{p}) \psi_{n, \mathbf{p}, \ell^{\prime}}^{(i)}=E_{n, \mathbf{p}}^{(i)} \psi_{n, \mathbf{p}, \ell}^{(i)} \quad(n=0,1, \cdots, \infty),
$$

with $i(=1,2,3,4)$ labeling the internal quantum number $\left(p, p^{*}\right) \otimes(\uparrow, \downarrow)$, where $p^{*}$ implies the $I_{3}=+1 / 2$ part of $N^{*}(1535)$. The total thermodynamic potential is now evaluated as

$$
\begin{aligned}
\Omega= & \sum_{n=0}^{\infty} \sum_{i=1}^{4} \int_{-f}^{f} \frac{d p_{z}}{\pi} \int \frac{d \mathbf{p}_{\perp}}{(2 \pi)^{2}}\left(E_{n, \mathbf{p}}^{(i)}-\mu_{B}^{*}\right) \theta\left(\mu_{B}^{*}-E_{n, \mathbf{p}}^{(i)}\right) \\
& -\frac{1}{2} m_{\omega}^{2} \omega_{0}^{2}-\frac{1}{2} \bar{\mu}^{2}\left(\delta \sigma^{2}+\sigma_{0}^{2}\right)+2 \sigma_{0}^{2} f^{2}+\frac{1}{4} \lambda_{4}\left[\left(\delta \sigma^{2}+\sigma_{0}^{2}\right)^{2}+2 \delta \sigma^{2} \sigma_{0}^{2}\right] \\
& -\frac{1}{6} \lambda_{6}\left[\left(\delta \sigma^{2}+\sigma_{0}^{2}\right)^{3}+6\left(\delta \sigma^{2}+\sigma_{0}^{2}\right) \delta \sigma^{2} \sigma_{0}^{2}\right]-m_{\pi}^{2} f_{\pi} \delta \sigma .
\end{aligned}
$$

\section{Parameter setting}

We treat $m_{0}$ as a free parameter. Parameters $g_{1}$ and $g_{2}$ can be determined by QCD vacuum property. Once $m_{0}$ is given, $m_{+}^{(0)}=939 \mathrm{MeV}$ and $m_{-}^{(0)}=1535 \mathrm{MeV}$ together with $\sigma_{0}=f_{\pi}=$ 92.2 $\mathrm{MeV}$ fix the values of $g_{1}$ and $g_{2}$ via

$$
m_{ \pm}^{(0)}=\frac{1}{2}\left[\sqrt{\left(g_{1}+g_{2}\right)^{2} \sigma_{0}^{2}+4 m_{0}^{2}} \mp\left(g_{1}-g_{2}\right) \sigma_{0}\right] .
$$

Table 1. Determined parameters for given chiral invariant mass

\begin{tabular}{c|ccccc}
\hline \hline$m_{0}$ & 500 & 600 & 700 & 800 & 900 \\
\hline$g_{1}$ & 9.03 & 8.49 & 7.82 & 7.00 & 5.97 \\
$g_{2}$ & 15.5 & 15.0 & 14.3 & 13.5 & 12.4 \\
$g_{\omega}$ & 11.3 & 9.13 & 7.30 & 5.66 & 3.52 \\
$\bar{\mu}[\mathrm{MeV}]$ & 441 & 437 & 406 & 320 & 114 \\
$\lambda_{4}$ & 42.2 & 40.6 & 35.7 & 23.2 & 4.47 \\
$\lambda_{6} \cdot f_{\pi}^{2}$ & 17.0 & 15.8 & 14.0 & 8.94 & 0.644 \\
\hline \hline
\end{tabular}




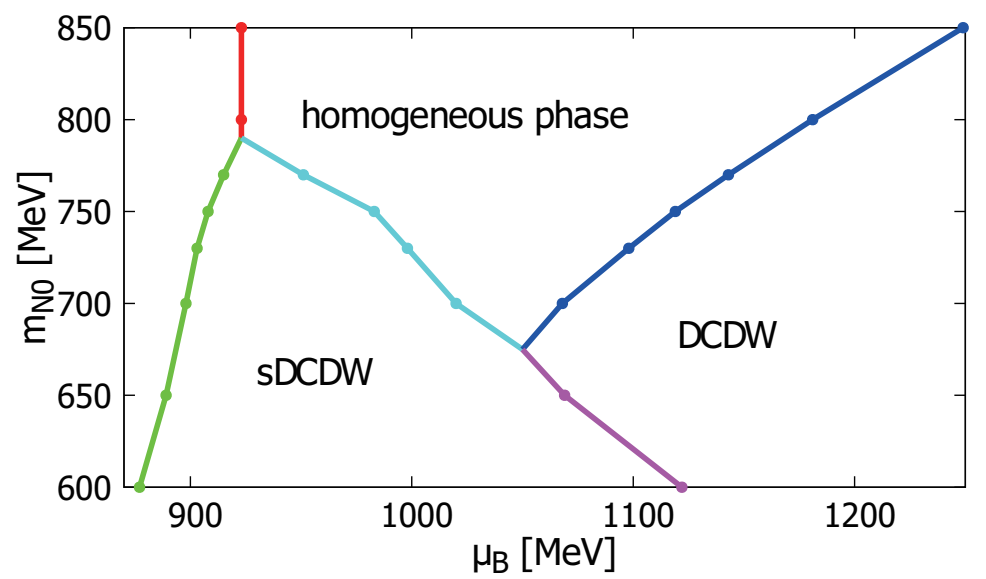

Figure 1. Phase structure in $\mu_{B}-m_{0}$ plane. Red line is $\mu_{B}=923 \mathrm{MeV}$ corresponding to the nuclear liquid-gas phase transition point.

From the stationary condition for $\sigma$ at vacuum, we have $\bar{\mu}^{2}=\lambda_{4} f_{\pi}^{2}-\lambda_{6} f_{\pi}^{4}-m_{\pi}^{2}$. Then we are left with three unknown parameters to be fixed, $\left\{\lambda_{4}, \lambda_{6}, g_{\omega}\right\}$. In order to fix these parameters, we use nuclear matter property at saturation density as done in $[17,19]$ :

$$
\begin{aligned}
& \rho_{0}=0.16 \mathrm{fm}^{-3}, \\
&\left.\left(\frac{\epsilon}{\rho}\right)\right|_{\rho=\rho_{0}}-m_{+}^{(0)}=\left.\mu_{B}^{*}\right|_{\rho=\rho_{0}}-m_{+}^{(0)}=-16 \mathrm{MeV}, \\
& K=\left.9 \rho_{0}^{2} \frac{\partial^{2}(\epsilon / \rho)}{\partial^{2} \rho}\right|_{\rho=\rho_{0}}=\left.9 \rho_{0} \frac{\partial P}{\partial \rho}\right|_{\rho=\rho_{0}}=240 \mathrm{MeV} .
\end{aligned}
$$

The first and second equations determine the value of $g_{\omega}$, and $\omega_{0}$ as a function of density, $\omega_{0}=g_{\omega} \rho / m_{\omega}^{2}$. The saturation condition $\left.\frac{\partial}{\partial \rho}(\epsilon / \rho)\right|_{\rho=\rho_{0}}=\left.P\right|_{\rho=\rho_{0}} / \rho_{0}^{2}=0$, and the condition for the incompressibility, the last equation of Eq. (8), together with the stationary condition $\left.\frac{\partial \Omega}{\partial \sigma}\right|_{\rho=\rho_{0}}=0$ determines parameters $\left\{\lambda_{4}, \lambda_{6}\right\}$ and the scalar condensate at saturation density, $\left.\sigma_{0}\right|_{\rho=\rho_{0}}$. The parameters are summarized in Table 1. As will be shown later, however, the saturated nuclear matter exists only as a metastable state once chiral invariant mass becomes smaller than some critical value, $m_{0} \lesssim 800 \mathrm{MeV}$.

\section{3 phase structure}

Figure 1 shows the phase structure in $\mu_{B}-m_{0}$ plane. We notice that there are two kinds of DCDW phase, "DCDW" and "sDCDW". Depending on the range where $m_{0}$ resides, qualitative picture of phase transitions changes.

For $m_{0} \gtrsim 780 \mathrm{MeV}$, there are three phases; (1) the vacuum phase for $\mu_{B} \leq 923 \mathrm{MeV}$, (2) the homogeneously chiral symmetry broken phase, and (3) the DCDW phase at high density. In the upper panel of Fig. 2, we show order parameters as a function of $\mu_{B}$ for $m_{0}=800 \mathrm{MeV}$. Just for comparison we also depict by magenta curve, the solution for the case where the condition $\delta \sigma=0$ is forced (that is, the case of the standard DCDW ansatz). 
(a)

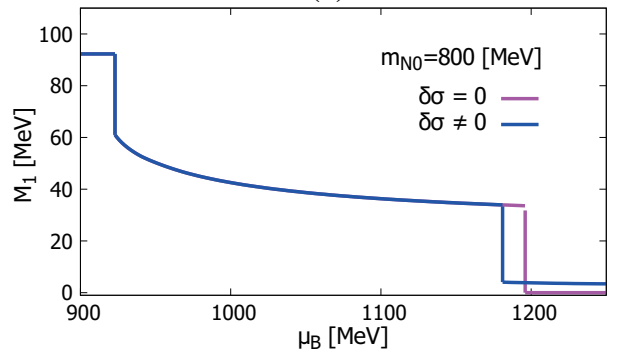

(c)

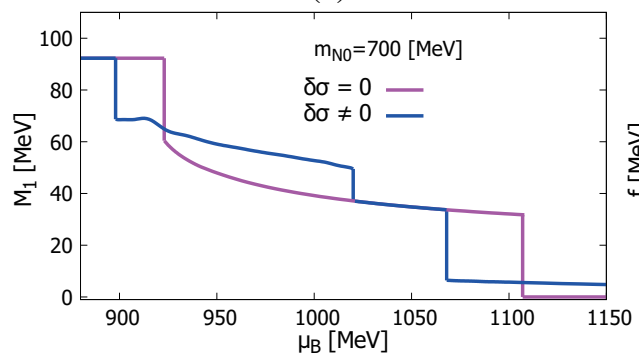

(b)

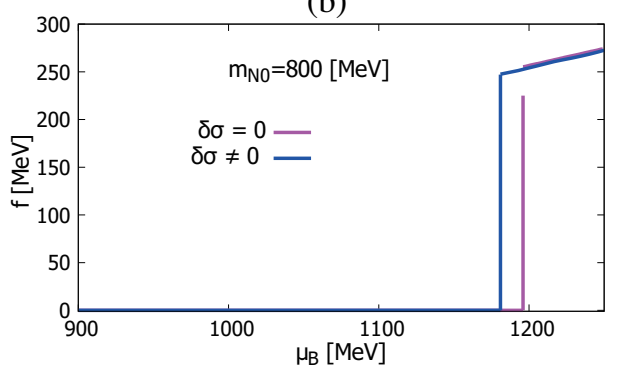

(d)

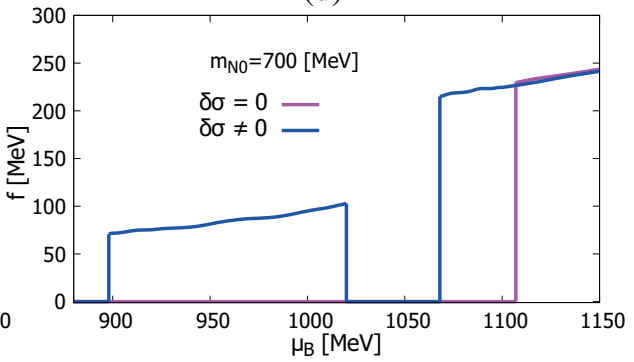

Figure 2. Left: the order parameter $M_{1}$ (see text) as a function of $\mu_{B}$ for $m_{0}=800 \mathrm{MeV}$ (upper panel) and that for $m_{0}=700 \mathrm{MeV}$ (lower panel). Right: the wavenumber $f$ as a function of $\mu_{B}$ for $m_{0}=800 \mathrm{MeV}$ (upper panel) and that for $m_{0}=700 \mathrm{MeV}$ (lower panel). Magenta curves correspond to the solutions obtained under the forced condition $\delta \sigma=0$.

Plotted in Fig. 2(a) is the space-averaged order parameter $M_{1}$, defined by

$$
M_{1} \equiv \frac{1}{2 V} \int_{-\infty}^{\infty} d^{3} x \operatorname{tr}[\langle M\rangle]= \begin{cases}\delta \sigma+\sigma_{0} & (f=0), \\ \delta \sigma & (f \neq 0),\end{cases}
$$

which provides a guide for the strength of chiral symmetry breaking. Figure 2(b) shows wavenumber $f$ as a function of $\mu_{B}$. Nonvanishing wavenumber $(f \neq 0)$ means the DCDW phase where the translational symmetry is also broken. We note that, when a shift $\delta \sigma$ is properly taken into account, the onset density the DCDW phase is brought to a lower density since it stabilizes the DCDW solution through the explicit symmetry breaking source, $-f_{\pi} m_{\pi}^{2} \delta \sigma$.

For $670 \mathrm{MeV} \lesssim m_{0} \lesssim 780 \mathrm{MeV}$, we find four phases. These are, going up in density, (1) the vacuum phase, (2) the "sDCDW" phase, (3) the homogeneously chiral-symmetry broken phase, and (4) the DCDW phase. What we call by "sDCDW" phase is the "shifted" DCDW phase, for a reason we will describe shortly. In order to see how phase transitions take place, we display in the lower panel of Fig. 2, the order parameters as a function of $\mu_{B}$ for $m_{0}=700 \mathrm{MeV}$. From these we see that, as the baryon density increases, the chiral symmetry restores through several steps. Moreover, we clearly see from the Fig. 2(d), that there are two DCDW phases; 'one at low density, and the other at high density, being separated by the homogeneously chiral-symmetry broken phase. We call the former "sDCDW", and the latter "ordinary" DCDW or simply DCDW.

Let us now have a closer look at the sDCDW which shows up next to the vacuum phase, in the region $900 \mathrm{MeV} \lesssim \mu_{B} \lesssim 1020 \mathrm{MeV}$ for $m_{0}=700 \mathrm{MeV}$. In contrast to the ordinary DCDW for $\mu_{B} \gtrsim 1070 \mathrm{MeV}$ the phase is not smoothly connected to the DCDW phase found at high density side for $m_{0}=800 \mathrm{MeV}$ as clearly seen in Fig. 1. In the ordinary DCDW, the magnitude of the wavenumber $f$ is much larger than $\delta \sigma$ (and also than $\sigma_{0}$ ). The chiral density 
wavelength is roughly $\lambda=\pi / f \sim 2-3 \mathrm{fm}$ which is still larger than averaged inter-nucleon spacing $\rho^{-1 / 3} \sim 1.2-1.3 \mathrm{fm}$. In the sDCDW phase, on the other hand, the wavenumber $f \sim 70-100 \mathrm{MeV}$ is comparable with $\delta \sigma \sim 50-70 \mathrm{MeV}$. The resulting chiral density wavelength $\lambda=\pi / f \sim 6-9 \mathrm{fm}$, which is much larger than the averaged inter-nucleon spacing $\rho^{-1 / 3} \sim 1-2 \mathrm{fm}$. Most interesting fact is that in the sDCDW phase the amplitude of condensate is smaller than the magnitude of the shift, namely, $\sigma_{0}<\delta \sigma$. Accordingly, the center of the chiral spiral in the $\left(\sigma, \pi^{0}\right)$ chiral plane, which is located near origin in the ordinary DCDW phase, is significantly shifted to the $\sigma$ direction. This is why we name the phase the "shifted" DCDW (abbreviated to "sDCDW") phase. We would like to stress that, on the contrary to the ordinary DCDW phase, $\delta \sigma$ in sDCDW phase is not due to the symmetry breaking source term, but rather spontaneously generated. This means, the solution to the stationary condition for $\delta \sigma$ would not vanish even in chiral limit.

As the chiral invariant mass $m_{0}$ is decreased, the density window for the homogeneously chiral-symmetry broken phase shrinks as the pressure of two kinds of DCDW phase gets stronger. And once the condition $m_{0} \lesssim 670 \mathrm{MeV}$ is met, it does no longer exist.

\section{Conclusions and outlook}

We studied the inhomogeneous phase structure in nuclear matter using a nucleon-based model with parity doublet structure where $N^{*}(1535)$ is introduced as the chiral partner of $N(939)$. Adopting an extended ansatz for DCDW, Eq. (4), we found that, the sDCDW phase shows up in addition to the ordinary DCDW phase when the value of chiral invariant mass $m_{0}$ is below some threshold, $\sim 780 \mathrm{MeV}$.

The ordinary DCDW phase appears at high density, where the space average of chiral condensate $M_{1}$, Eq. (9), is typically less than $10 \mathrm{MeV}$. This implies that this phase smoothly transforms into the standard DCDW with $\delta \sigma=0$ as the chiral limit is approached. The wavenumber $f$ has value of $200 \sim 300 \mathrm{MeV}$, corresponding to the density wavelength $\lambda \sim 2$ $-3 \mathrm{fm}$, which is in fair agreement with the result obtained in [20].

On the other hand, when $m_{0} \lesssim 780 \mathrm{MeV}$, the sDCDW phase appears at low density. This phase is characterized by a smaller wavenumber $f$ and a large shift of chiral condensate, $\delta \sigma$. It is noteworthy that it is not the effect of explicit chiral symmetry breaking but the dynamical symmetry breaking that produces this large shift of chiral condensate. So we expect that this sDCDW phase survives in the chiral limit.

The parameter range of chiral invariant mass where the sDCDW is stabilized, fails to realize nuclear matter as the pressure of sDCDW is so strong that it washes out the liquidgas phase transition structure. Then, one might think that the present model for $m_{0}$ less than $780 \mathrm{MeV}$ is ruled out. However, the chiral invariant mass $m_{0}$ can have density dependence as in Ref. [20] which shows that $m_{0}$ decreases against increasing density. In such a case, the sDCDW phase may be realized in high density nuclear matter in the real world.

Exploring the elementary excitations in the sDCDW phase deserves further investigations in future. In the ordinary DCDW phase, a particular combination of the space translation and chiral rotation remains unbroken $[21,22]$. As a result, there is no extra Nambu-Goldstone boson other than three pions. In contrast, there is no such unbroken combination of symmetry in the sDCDW phase. We then expect that a phonon mode appears in the sDCDW phase which may signal the phase. An inclusion of external magnetic field may provide another interesting direction of extending current work. For quark matter, several studies were already devoted to the topic of inhomogeneous phases under the magnetic field [23-27]. The analysis within the nucleon-based model with including both $\delta \sigma$ and magnetic field would be an interesting subject worth exploring. 
Acknowledgement. This work was partially supported by JPSP KAKENHI Grant Number JP16K05346 (H.A.) and 16K05345 (M.H.).

\section{References}

[1] E. Nakano and T. Tatsumi, Phys. Rev. D 71, 114006 (2005).

[2] See for a review, M. Buballa and S. Carignano, Prog. Part. Nucl. Phys. 81, 39 (2015).

[3] D. Nickel, Phys. Rev. D 80, 074025 (2009).

[4] S. Carignano, D. Nickel and M. Buballa, Phys. Rev. D 82, 054009 (2010).

[5] S. Karasawa and T. Tatsumi, Phys. Rev. D 92, 116004 (2015).

[6] P. Adhikari, J. O. Andersen and P. Kneschke, Phys. Rev. D 96, 016013 (2017).

[7] D. Nickel, Phys. Rev. Lett. 103, 072301 (2009).

[8] H. Abuki, D. Ishibashi and K. Suzuki, Phys. Rev. D 85, 074002 (2012).

[9] H. Abuki, Phys. Lett. B 728, 427 (2014).

[10] S. Carignano, M. Mannarelli, F. Anzuini and O. Benhar, Phys. Rev. D 97, 036009 (2018).

[11] T. G. Lee, K. Nishiyama, N. Yasutake, T. Maruyama and T. Tatsumi, JPS Conf. Proc. 14, 020808 (2017).

[12] Y. Takeda, H. Abuki and M. Harada, Phys. Rev. D 97, 094032 (2018).

[13] C. E. Detar and T. Kunihiro, Phys. Rev. D 39, 2805 (1989).

[14] D. Jido, M. Oka and A. Hosaka, Prog. Theor. Phys. 106, 873 (2001).

[15] M. Bando, T. Kugo and K. Yamawaki, Phys. Rept. 164, 217 (1988).

[16] M. Harada and K. Yamawaki, Phys. Rept. 381, 1 (2003).

[17] Y. Motohiro, Y. Kim and M. Harada, Phys. Rev. C 92, 025201 (2015); Erratum: [Phys. Rev. C 95, 059903 (2017)].

[18] See for example, D. Nickel and M. Buballa, Phys. Rev. D 79, 054009 (2009).

[19] Y. Takeda, Y. Kim and M. Harada, Phys. Rev. C 97, 065202 (2018).

[20] A. Heinz, F. Giacosa and D. H. Rischke, Nucl. Phys. A 933, 34 (2015).

[21] T. G. Lee, E. Nakano, Y. Tsue, T. Tatsumi and B. Friman, Phys. Rev. D 92, 034024 (2015).

[22] Y. Hidaka, K. Kamikado, T. Kanazawa and T. Noumi, Phys. Rev. D 92, 034003 (2015).

[23] I. E. Frolov, V. C. Zhukovsky and K. G. Klimenko, Phys. Rev. D 82, 076002 (2010).

[24] K. Nishiyama, S. Karasawa and T. Tatsumi, Phys. Rev. D 92, 036008 (2015).

[25] R. Yoshiike, K. Nishiyama and T. Tatsumi, Phys. Lett. B 751, 123 (2015).

[26] G. Cao and A. Huang, Phys. Rev. D 93, 076007 (2016).

[27] H. Abuki, Phys. Rev. D 98, 054006 (2018); JPS Conf. Proc. 20, 011017 (2018); EPJ Web Conf. 129, 00036 (2016). 